\title{
Reflective Fiber Surface Plasmon Resonance Sensor for High-Sensitive Mercury Ion Detection
}

\author{
Zhenlin Chen, Kunlin Han and Ya-Nan Zhang *D \\ College of Information Science and Engineering, Northeastern University, Shenyang 110819, China; \\ aschenzhenlin@163.com (Z.C.); 13518307975@163.com (K.H.) \\ * Correspondence: zhangyanan@ise.neu.edu.cn
}

Received: 10 February 2019; Accepted: 29 March 2019; Published: 9 April 2019

check for updates

\begin{abstract}
This paper proposes a reflective fiber mercury ion sensor based on the surface plasmon resonance (SPR) principle and chitosan (CS)/polyacrylic acid (PAA) multilayer sensitive film. By optimizing the coating parameters of the gold film, the refractive index (RI) sensitivity of the reflective SPR sensor is demonstrated to be $2110.33 \mathrm{~nm} / \mathrm{RIU}$. Then, a multi-layer CS/PAA film is fixed on the surface of the gold film as a mercury ion sensitive film to form a reflective SPR fiber mercury ion sensor. Experimental results demonstrate that the sensor can be used to detect different concentrations of mercury ions with a high sensitivity of $0.5586 \mathrm{~nm} / \mu \mathrm{M}$ and good specificity and repeatability. Therefore, the reflective SPR fiber mercury ion sensor shows great promise for future applications of environmental monitoring and drinking water safety.
\end{abstract}

Keywords: mercury ion; fiber optic sensor; surface plasmon resonance; reflective sensor

\section{Introduction}

The problem of heavy metal pollution is becoming more and more serious and has always been one of the key issues of environmental pollution [1,2]. Mercury, as one of the heavy metal contaminants, causes serious health issues, affecting neurological, renal organ, and gastrointestinal systems [3]. The main form of mercury as a pollutant is mercury ion $\left(\mathrm{Hg}^{2+}\right)$, which has high chemical activity in solution [4]. Therefore, a reliable and convenient $\mathrm{Hg}^{2+}$ detection method is of great significance for the safety of drinking water. There are many traditional methods for $\mathrm{Hg}^{2+}$ detection, such as those based on colorimetry and fluorescence [5,6], nanoparticles [7] or functional polymers [8]. These methods typically require time-consuming assay procedures and complex specialized laboratory equipment and samples.

In contrast, fiber optic sensors have unique advantages, such as multiplexing, high flexibility, high sensitivity, remote sensing, low cost, and immunity to electromagnetic interference [9-11], which play an increasingly important role in the detection of heavy metal ions. In recent years, researchers proposed many fiber sensing technologies to detect heavy metal ions, including: Evanescent wave absorption [12], Mach-Zehnder interferometer [13], fluorescence [14], and surface plasmon resonance (SPR) [15]. Compared with other methods, the optical fiber SPR sensor has higher sensitivity. It has been reported that fiber optic SPR sensors have been used to detect the concentration of manganese ions [16], cadmium ions [17], and mercury ions [18] in solution. However, the sensitive films of the presented mercury ion sensors usually rely on gold nanoparticles, the use of which does not ensure uniformity of the film, and thus affecting the linearity and even measurement accuracy of such sensors $[18,19]$. Besides, the sensing heads of the present sensors often work in the transmission type $[18,20]$, which is not convenient for the insertion of the sensing head into the analysis solution and not suitable for remote measurement of this kind of sensor. It has been demonstrated that fiber SPR sensors could also work in the reflective type by coating an Au mirror [21] or Ag mirror [18] on 
the fiber end. However, the additional coating process of the metal film on the fiber end increases the fabrication complexity of the sensing head. Besides, the Ag film is easily oxidized, which then influences the reflectivity.

In order to solve the above problems, this paper proposes a reflective fiber SPR sensor for measuring $\mathrm{Hg}^{2+}$ concentrations. The sensor head is fabricated by splicing a single mode fiber (SMF) and a multimode fiber (MMF). A gold film for exciting SPR and a gold mirror for reflecting optical resonance are simultaneously coated on the surface of the SMF by using a sputtering apparatus. This reflective sensing structure can be used as a plug-in probe and is suitable for remote measurement. Also, a multilayered chitosan (CS)/polyacrylic acid (PAA) was successfully and uniformly coated on the side surface of the gold film, which was demonstrated as a low cost and excellently sensitive film for the detection of the $\mathrm{Hg}^{2+}$ concentrations [20]. When $\mathrm{Hg}^{2+}$ is adsorbed on the sensitive film, the refractive index of the sensitive film changes with the $\mathrm{Hg}^{2+}$ concentration to affect the resonant peak wavelength of the SPR. In addition, the fiber optic sensor designed in this paper has relatively good recyclability and stability while being simple to manufacture and operate.

\section{Simulation and Experiment}

\subsection{Preparation of Optical Fiber SPR Sensing Probe}

As shown in Figure 1, the reflective fiber SPR $\mathrm{Hg}^{2+}$ sensor is made of an SMF (with a core diameter of $8.2 \mu \mathrm{m}$, cladding diameter of $125 \mu \mathrm{m}$, and length of $1 \mathrm{~cm}$ ) and an MMF (with a core diameter of $62.5 \mu \mathrm{m}$ and cladding diameter of $125 \mu \mathrm{m}$ ). The difference in the core diameters between the MMF and the SMF makes the light leak into the cladding of the SMF. When the leaked light is reflected at the boundary of the cladding region and the surrounding environment under conditions of total internal reflection, the light wave induces the optical evanescent wave on the surface of the SMF. A gold thin film for exciting SPR is coated on the surface of the SMF, whose coating layer is removed. Further, the surface of the gold film of the SMF portion is coated with an $\mathrm{Hg}^{2+}$ sensitive film. When light enters the SMF from the MMF, a portion of the light enters the cladding of the SMF, producing a large number of cladding modes. The coupling mode and surface plasmon wave couples in accordance with the phase matching conditions, resulting in an SPR spectrum.

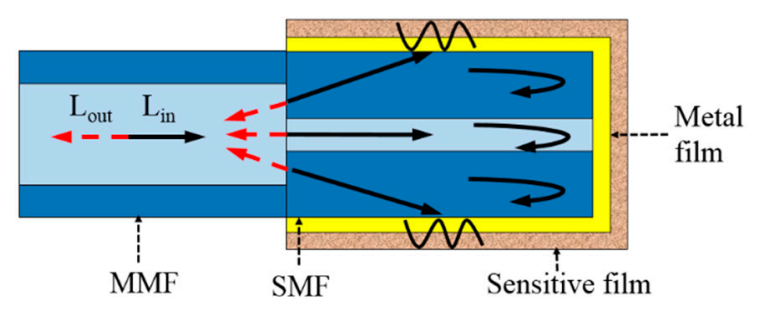

Figure 1. Schematic diagram of the sensing principles.

It is well known that the SPR spectrum depends on the surrounding refractive index, incident angle of light (which is a fixed value for this proposed sensor), and thickness and length of the Au film. By using the simulation model proposed in [22], Figure 2 shows the simulation results of SPR spectra along with the variations of the gold film length, gold film thickness, and refractive index around the gold film. It can be seen from Figure $2 a$ that the length of the gold film has no influence on the wavelength position of the resonance valley, but has a large influence on the shape of the resonance spectrum. On the other hand, the thickness of the gold film influences both the wavelength position and the shape of the resonance spectrum (see Figure $2 b$ ), and even makes the SPR valley disappear. When the length and thickness of the gold film are set as $10 \mathrm{~mm}$ and $40 \mathrm{~nm}$ (the optimum parameters), a change of the SPR spectra along with the refractive index variation can be obtained as shown in Figure 2c. As seen, the valley of the SPR spectrum generates an obvious red shift with the increase of the refractive index. Since the $\mathrm{Hg}^{2+}$ sensitive film is coated on the surface of the metal film, 
the refractive index around the metal film changes with the variation of the $\mathrm{Hg}^{2+}$ concentration due to adsorption of $\mathrm{Hg}^{2+}$, which will then affect the SPR resonance wavelength and intensity. The optical signal carrying the resonant wavelength is reflected back to the MMF, and the SPR wavelength in the output spectrum shifts. Therefore, the change in the resonant wavelength of the output spectrum can reflect the information of the $\mathrm{Hg}^{2+}$ concentration in the measured solution.

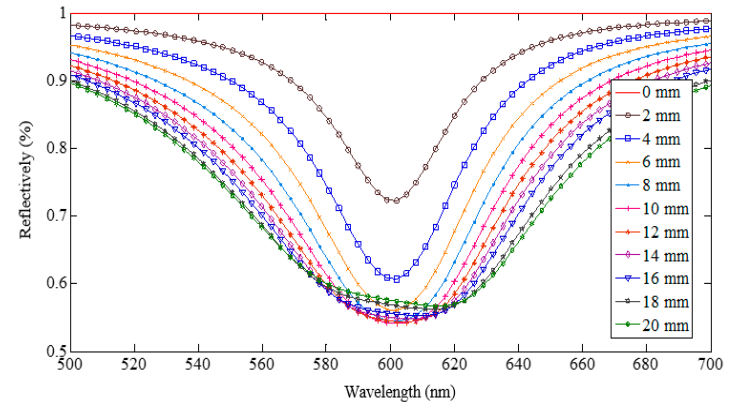

(a)

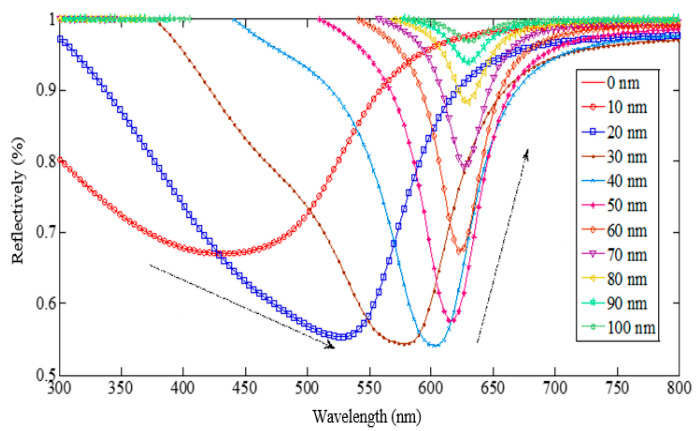

(b)

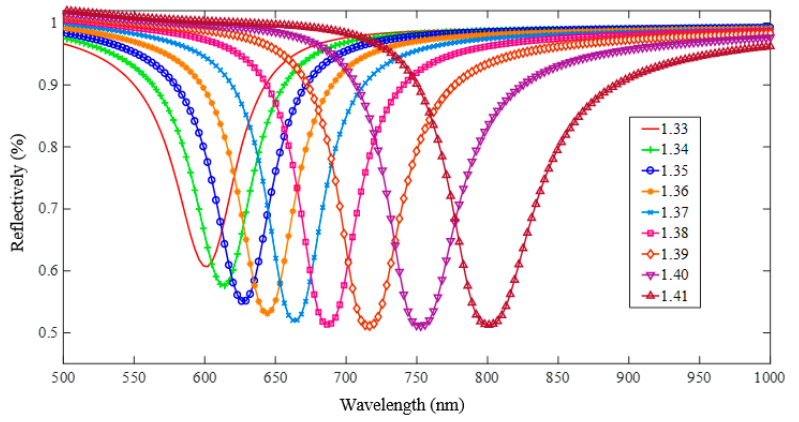

(c)

Figure 2. Variation of the simulated SPR spectra along with the changes of the gold film length (a), gold film thickness (b), and surrounding refractive index (c).

In this paper, Au film was coated on the surface of the fiber by using a JS-1600 ion sputtering apparatus. From the above simulation analyses, we know that the SPR spectrum depends on the length and thickness of the gold film. For our fabrication experiment of the sensing head, the length of the gold film can be controlled by adjusting the length of the SMF. However, the thickness of the gold film depends on both the coating current and the coating time of the ion sputtering. Besides, according to the simulation results of the SPR spectrum and our practical experiences, the thickness of the lateral $\mathrm{Au}$ film should be around $40 \mathrm{~nm}$ to effectively excite SPR while the thickness of the terminal Au film should be larger than $80 \mathrm{~nm}$ to ensure efficient reflectivity on the fiber end. To meet this requirement, we placed the SMF vertically in the JS- 1600 ion sputtering apparatus-namely, the end face of the SMF without coating faces the Au target - to ensure that the thickness of the terminal Au film is larger than that of the lateral Au film. After a series of experiments, it was verified that the thicknesses of both $\mathrm{Au}$ films met this requirement in a simultaneous sputtering process, by only controlling the coating time and coating current. To investigate the coating rule and to obtain a good SPR spectrum, several optical fiber sensing probes with different coating currents and coating times were fabricated and then their SPR spectra were measured by immersing these probes in pure water with a fixed refractive index of 1.3321. The lengths of the SMF in these sensing probes were all controlled to be $10 \mathrm{~mm}$. Figure 3 shows the reflection spectra of the fiber sensor after Au was plated in different coating current conditions. These were a coating time of $90 \mathrm{~s}$, and coating currents were selected as $2 \mathrm{~mA}, 4 \mathrm{~mA}, 6 \mathrm{~mA}$, and $8 \mathrm{~mA}$, respectively; the experimental results are shown in Figure 2a. It can be seen that when the current was selected to be $6 \mathrm{~mA}$, the obtained reflection spectrum has the largest resonance depth 
and the minimum half width. On the other hand, the coating current was $6 \mathrm{~mA}$, and the coating time was selected to be $60 \mathrm{~s}, 75 \mathrm{~s}, 90 \mathrm{~s}$, and $105 \mathrm{~s}$, respectively; these experimental results are shown in Figure $3 \mathrm{~b}$. It can be seen that when the coating time was $90 \mathrm{~s}$, the obtained reflection spectrum has the largest resonance depth and the minimum half width. According to the above experimental results, the optimal experimental parameters for the Au plating film were $6 \mathrm{~mA}$ for the coating current and $90 \mathrm{~s}$ for the coating time. By comparing the optimum experiment result shown in Figure $3 \mathrm{~b}$ (the blue line corresponds to a length of $10 \mathrm{~mm}$, refractive index of 1.3321, coating current of $6 \mathrm{~mA}$, and coating time of $90 \mathrm{~s}$ ) and the optimum simulation result shown in Figure 2c (the red line corresponds to an $\mathrm{Au}$ length of $10 \mathrm{~mm}$, refractive index of 1.33, Au thickness of $40 \mathrm{~nm}$ ), we can suppose that the thickness of the $\mathrm{Au}$ film under the optimal experimental parameters is around $40 \mathrm{~nm}$. Figure 4 shows micrographs of the fiber sensing probe before and after Au coating at this optimal parameter, which demonstrated that the Au film is coated well on the surface of the fiber by using the above method.

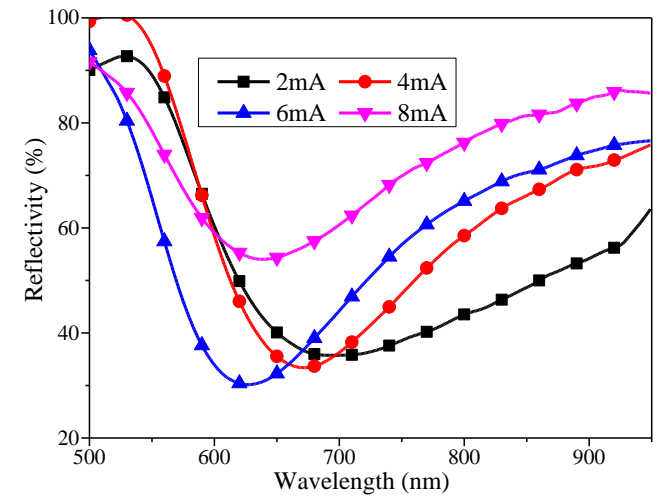

(a)

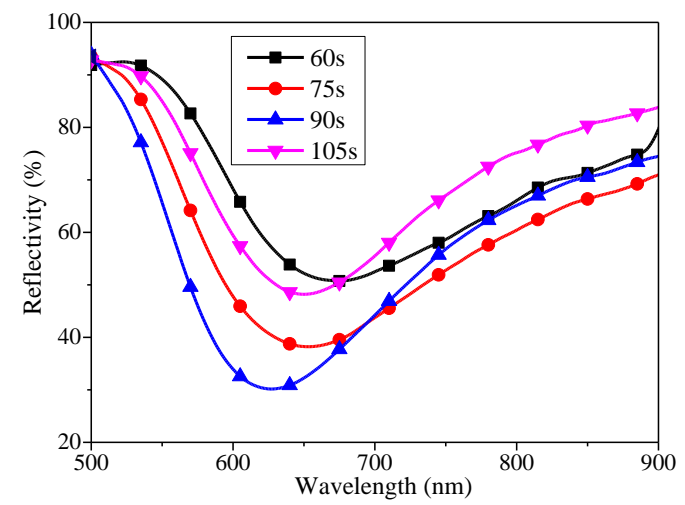

(b)

Figure 3. Experimental SPR spectra when the Au film is coated with different coating currents (a) and coating times $(\mathbf{b})$.
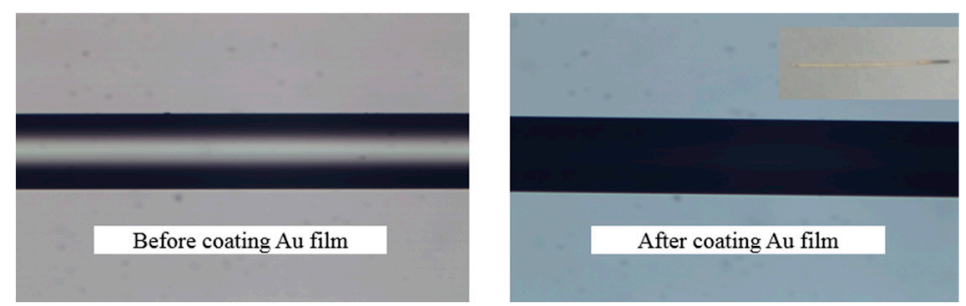

Figure 4. Optical fiber surface micrographs before and after Au film coating.

\subsection{Preparation and Coating of Mercury Ion Sensitive Film}

Chitosan (CS)/ polyacrylic acid (PAA) was selected as the sensitive film for $\mathrm{Hg}^{2+}$ sensing. During the sensitive film coating process, the surface of the Au film was subjected to activation-COOH treatment so that the CS/PAA sensitive film was stably fixed on the Au film. As shown in Figure 5, the $\mathrm{Au}$ film surface activation- $\mathrm{COOH}$ treatment step and the CS/PAA sensitive film coating were as follows: (1) The sensor probe was thoroughly cleaned by using anhydrous ethanol and distilled water in turn; (2) the sensor probe was inserted into mercapto undecanoic acid (MUA) solution with a concentration of $50 \mathrm{mmol} / \mathrm{L}$ for one day at room temperature conditions, so the -HS in the MUA molecule combined with the Au film to form a stable Au-S bond, and the -HS and -COOH were modified on the surface of the Au film; (3) the sensor probe was immersed in a mixed solution, which consisted of $0.4 \mathrm{~mol} / \mathrm{L}$ EDC (1-ethyl-(3-dimethylaminopropyl) carbodiimide hydrochloride) and $0.1 \mathrm{~mol} / \mathrm{L}$ NHS (N-hydroxysuccinimide) at a ratio of 1:1 for one hour at room temperature, which activated the - $\mathrm{COOH} ;(4)$ the sensor probe was attached to a dip coater, and then immersed in a $2 \% \mathrm{CS}$ solution at a speed of $100 \mathrm{~mm} / \mathrm{min}$, then allowed to stand for $7 \mathrm{~min}$, and finally pulled out from the CS 
solution at a speed of $100 \mathrm{~mm} / \mathrm{min}$, during which the $-\mathrm{COOH}$ on the surface of the Au film coupled with $-\mathrm{NH}_{2}$ in the CS to adsorb the CS molecule onto the $\mathrm{Au}$; (5) the sensor probe was immersed in an $8 \%$ PAA solution at a speed of $100 \mathrm{~mm} / \mathrm{min}$ and stand for $7 \mathrm{~min}$, and then pulled out at a rate of $100 \mathrm{~mm} / \mathrm{min}$, during which the PAA solution was absorbed; (6) steps (4) and (5) were repeated until an appropriate number of layers of CS/PAA sensitive film were coated on the surface of the Au film; (7) the sensor probe coated with the sensitive film was placed in a $60^{\circ} \mathrm{C}$ incubator for $12 \mathrm{~h}$ to completely dry the sensitive film and fix it on the probe surface.

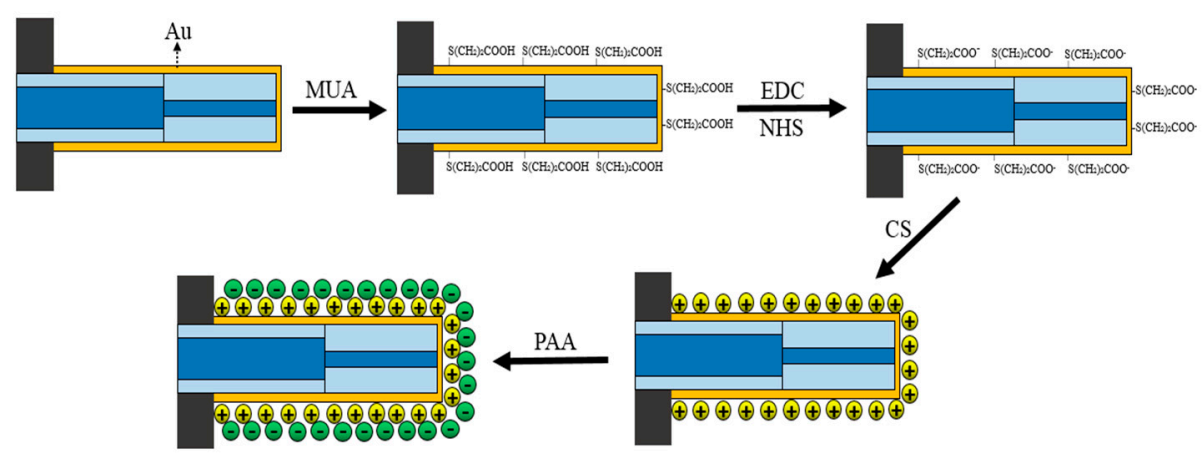

Figure 5. Coating process of the $\mathrm{Hg}^{2+}$ sensitive film.

\subsection{Experimental Device}

The measurement system of the reflective fiber SPR $\mathrm{Hg}^{2+}$ sensor is shown in Figure 6, which consists of a halogen light source, a Y-type fiber coupler, a marine spectrometer, a computer, and a reflective SPR fiber sensor head. Light is emitted from the light source and transmitted to the sensing area through one port of the Y-type fiber. The sensor probe carries the $\mathrm{Hg}^{2+}$ concentration information, which enters the spectrometer through another port of the Y-type fiber. Finally, the $\mathrm{Hg}^{2+}$ concentration can be obtained by demodulating the output spectrum shown on the computer.

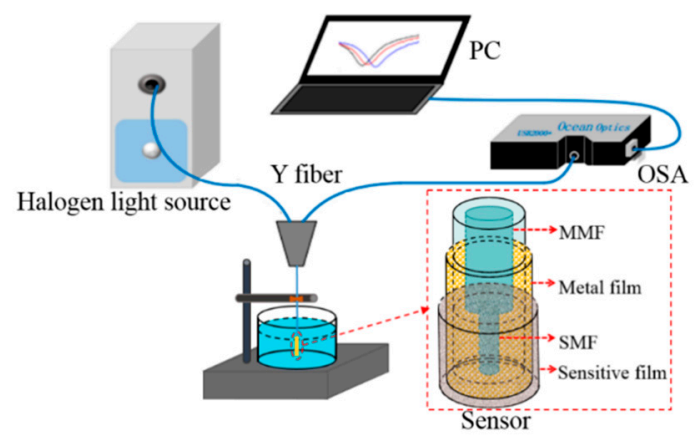

Figure 6. Reflective fiber SPR $\mathrm{Hg}^{2+}$ measurement system.

\section{Performance Test}

\subsection{Refractive Index Measurement Experiment}

A fiber SPR sensor can detect the change of the external refractive index, which is the basic measurement principle of an $\mathrm{Hg}^{2+}$ sensor. Therefore, we first carried out the refractive index measurement experiment of the reflective fiber SPR sensor before coating the CS/PAA sensitive film. As shown in Figure 7, when the external refractive index increases from 1.3333 to 1.3787, the resonant dip of the SPR moves towards the long wavelength direction. From the fitting result shown in Figure 7b, the refractive index sensitivity of the SPR sensor reaches $2110.33 \mathrm{~nm} / \mathrm{RIU}$ and the coefficient of determination, $R^{2}$ (also known as goodness of fit), is 0.9798 . The maximum value of $R^{2}$ is 1 , and the closer the $R^{2}$ gets to 1 , the better the linearity of the fitting curve. The experiment results are consistent with the simulation results shown in Figure 2c, which demonstrates the feasibility of the 
fabricated sensor in high-sensitive refractive index sensing. In addition, combined with the sensitive properties of the CS/PAA sensitive film, we can conclude that the proposed sensor can be used for the sensing of $\mathrm{Hg}^{2+}$ concentrations. Therefore, in subsequent experiments, we will investigate and analyze the performances of the proposed sensor in the measurement of $\mathrm{Hg}^{2+}$ concentrations.

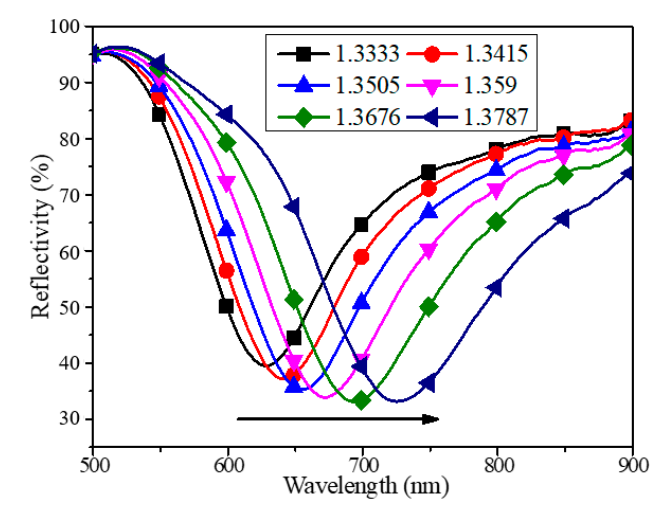

(a)

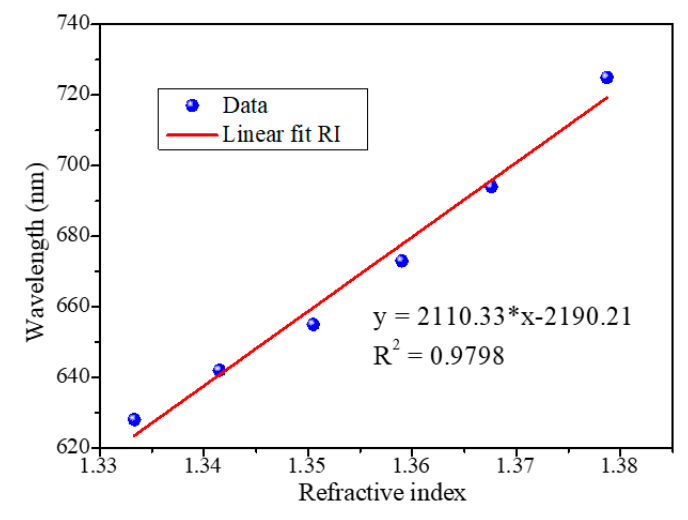

(b)

Figure 7. Resonant spectra (a) and linear fitting result (b) of a reflective fiber SPR sensor in refractive index measurement.

\subsection{Optimization of the Number of Layers of $\mathrm{Hg}^{2+}$ Sensitive Film}

Under the same conditions, fiber reflective SPR sensors with $\mathrm{Hg}^{2+}$ sensitive films of 5 layers, 10 layers, and 15 layers were fabricated, named as $(\mathrm{CS} / \mathrm{PAA})_{5},(\mathrm{CS} / \mathrm{PAA})_{10}$, and (CS/PAA $)_{15}$, respectively. These fiber sensors were immersed in $50-\mu \mathrm{M} \mathrm{Hg}^{2+}$ solution, and the output spectra were recorded with the detection time, respectively, as shown in Figure 8. From the experimental results, it can be seen that with the increase of the detection time, the spectral wavelength moves to the long wavelength direction, and finally stops moving. This is because the adsorption of $\mathrm{Hg}^{2+}$ leads to an increase of the cross-linking degree and an increase of the refractive index of the sensitive film. The wavelength shifts at equilibrium are $15 \mathrm{~nm}, 20 \mathrm{~nm}$, and $6 \mathrm{~nm}$, respectively. When measuring the same concentration of $\mathrm{Hg}^{2+}$ solution, the (CS/PAA) 10 sensor has the largest spectral wavelength shift, namely the highest sensitivity. Therefore, the optimal number of layers of the sensitive film was selected as 10 in the following experiments.

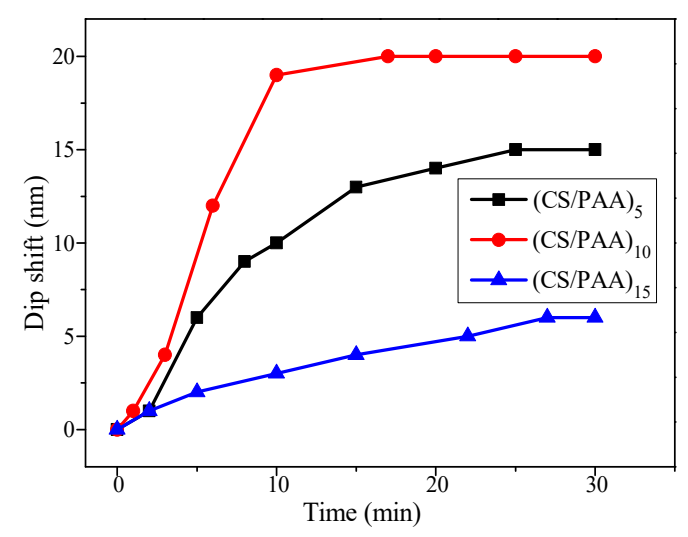

Figure 8. Comparison of wavelength shifts for SPR sensors with different numbers of layers of sensitive film.

\subsection{Measurement Experiment of Mercury Ion Concentration}

In order to test the sensing characteristics of the reflective SPR $\mathrm{Hg}^{2+}$ sensor, a series of $\mathrm{Hg}^{2+}$ solutions with different concentrations were prepared by diluting $1 \mathrm{mmol} / \mathrm{L}$ standard solution of $\mathrm{Hg}^{2+}$ 
with ultra-pure water. Figure 9a shows the change of the wavelength shift with time when detecting different concentrations of $\mathrm{Hg}^{2+}$ solution. When the detection time reached about $20 \mathrm{~min}$, the spectrum stopped changing and reached stability. A linear fitting relationship between the spectral wavelength shift and $\mathrm{Hg}^{2+}$ concentration is shown in Figure 9b. With the increase of the $\mathrm{Hg}^{2+}$ concentration, the wavelength shift increases when the spectrum finally reaches stability. The sensitivity of the sensor is $0.5586 \mathrm{~nm} / \mu \mathrm{M}$ in the concentration range of 0 to $30 \mu \mathrm{M}, 0.1239 \mathrm{~nm} / \mu \mathrm{M}$ in the concentration range of 30 to $100 \mu \mathrm{M}$, and $0.02 \mathrm{~nm} / \mu \mathrm{M}$ in the concentration range of 100 to $200 \mu \mathrm{M}$. From the experimental results, it can be seen that the concentration sensitivity of the sensor decreases with the increase of the $\mathrm{Hg}^{2+}$ concentration in the solution. This is because the chelating sites of $\mathrm{Hg}^{2+}$ in the sensing film gradually reach saturation, and the refractive index of the sensing layer gradually reaches the maximum value.

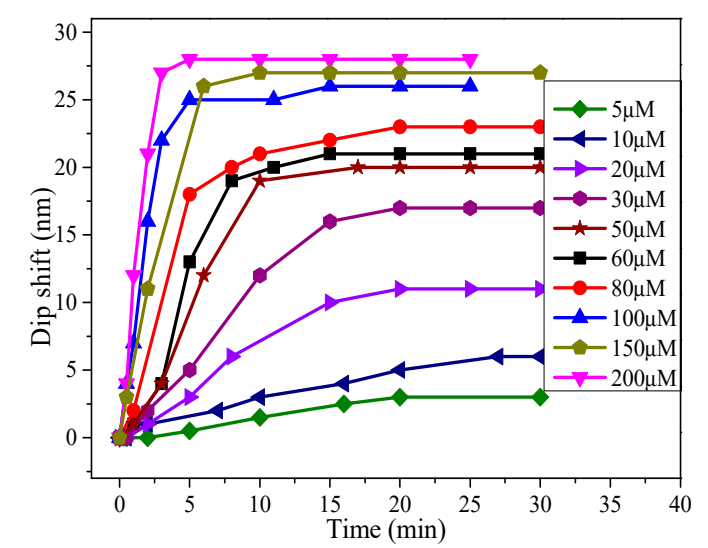

(a)

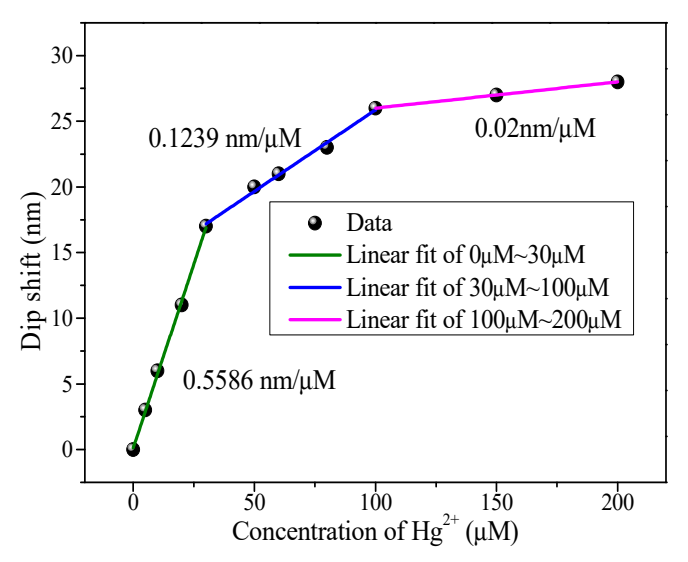

(b)

Figure 9. Resonant spectra (a) and linear fitting result (b) of the reflective fiber SPR sensor in the $\mathrm{Hg}^{2+}$ concentration measurement.

\subsection{Specificity Analysis}

Since CS and PAA were the sensitive materials chosen in this experiment, the $-\mathrm{NH}_{2}$ and $-\mathrm{COOH}$ in the molecule can form chelates with most metal ions. Therefore, the sensitive film can not only adsorb $\mathrm{Hg}^{2+}$, but also other metal ions, such as $\mathrm{K}^{+}, \mathrm{Na}^{+}, \mathrm{Cu}^{2+}, \mathrm{Ag}^{+}$, and $\mathrm{Fe}^{3+}$. In order to investigate the specificity of the fiber SPR $\mathrm{Hg}^{2+}$ sensor, $\mathrm{K}^{+}, \mathrm{Na}^{+}, \mathrm{Cu}^{2+}, \mathrm{Ag}^{+}$, and $\mathrm{Fe}^{3+}$ solutions with an ion concentration of $50 \mu \mathrm{M}$ were prepared, and then solutions containing different ions with the same concentration were measured by the SPR sensor. The experimental results are shown in Figure 10. As can be seen from Figure 10a, with the increase of the detection time, the wavelength shift increases gradually with the detection time, and finally reaches stability. It can be seen from Figure 10b that the stable wavelength shift of $\mathrm{Hg}^{2+}$ is obviously larger than that of the other ions. Therefore, this proposed SPR sensor shows good specificity to $\mathrm{Hg}^{2+}$.

\subsection{Repeatability Analysis}

Figure 11a shows the wavelength shifts of the resonant spectra during the detection of $\mathrm{Hg}^{2+}$ solutions with different concentrations. After each detection of the $\mathrm{Hg}^{2+}$ concentration, the sensor was immersed in dilute hydrochloric acid solution with $\mathrm{pH}=3.1$. After about $10 \mathrm{~min}$, the tracking wavelength could be basically restored to its original position. It can be seen that when the sensor was immersed in solution of different concentrations, the spectral wavelength shifts differently. The main reason is that when the $\mathrm{pH}$ of the tested solution was less than 4 , the $-\mathrm{NH}_{2}$ and $-\mathrm{COOH}$ in the sensitive materials were protonated, resulting in the release of $\mathrm{Hg}^{2+}$ bound to it, which guarantees the regeneration and reuse of the sensor. Experimental results show that the sensor has no large loss after 10 times being reused and shows good repeatability performance. The same sensor was also used to 
detect $\mathrm{Hg}^{2+}$ solution of a $50 \mu \mathrm{M}$ concentration with five cycles in a month. In each cycle, the sensor was firstly inserted into the $\mathrm{Hg}^{2+}$ solution of a $50 \mu \mathrm{M}$ concentration until the resonant wavelength reached equilibrium, and then the sensor was immersed in dilute hydrochloric acid solution with $\mathrm{pH}=3.1$ until the resonant wavelength recovered to the original wavelength. Figure $11 \mathrm{~b}$ shows the experimental results. In each cycle, the wavelength shifts of the sensor at equilibrium were $20 \mathrm{~nm}$, $20 \mathrm{~nm}, 21 \mathrm{~nm}, 19 \mathrm{~nm}$, and $21 \mathrm{~nm}$, respectively. A small difference between the measurement results of the five cycles exists. Besides, the rising velocities of the five cycles are different. It was apparent that there was a small difference between the measurement results of the five cycles. Besides, the rising velocities of the five cycles were different. As the long internal time between the first cycle and the fifth cycle (30 days), the potential influencing factor may be the temperature disturbance (the temperature variation in our lab may be $10^{\circ} \mathrm{C}$ ), which will influence the response velocity of the sensor. Besides, the variation of temperature will change the physicochemical properties of the CS/PAA sensitive film and the sensing properties of the SPR sensor, which will then induce measurement error in the mercury ion sensor. To solve this temperature crosstalk problem, one feasible method is to measure the mercury ion concentration and temperature simultaneously, and then compensate the influence of temperature $[23,24]$. We will focus on this problem in the future.

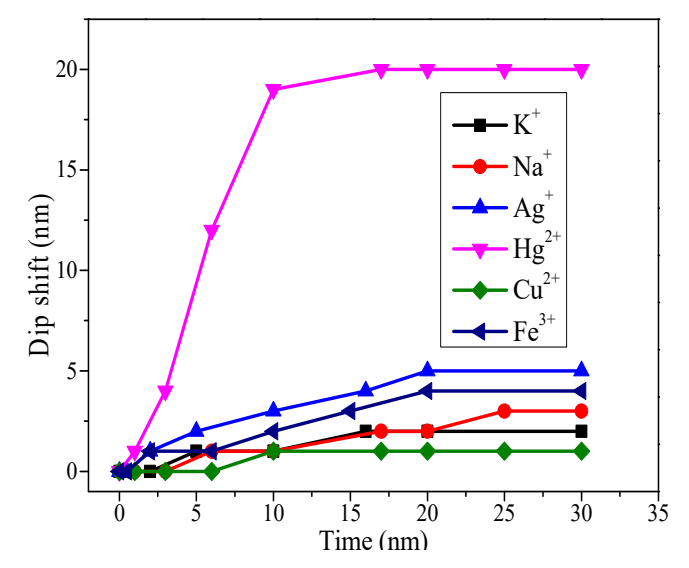

(a)

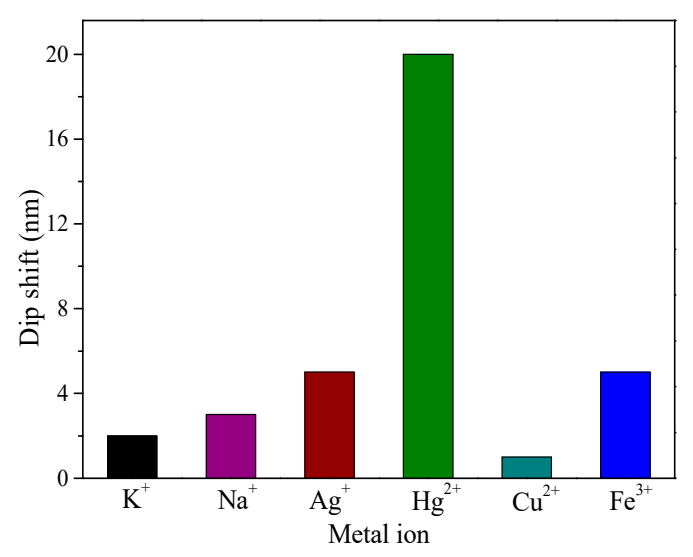

(b)

Figure 10. Resonant spectra (a) and wavelength shifts (b) of the reflective fiber SPR sensor in different metal ions.

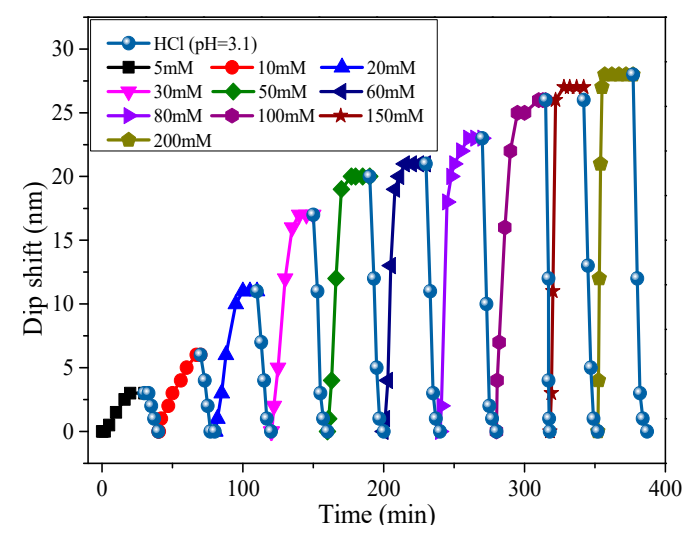

(a)

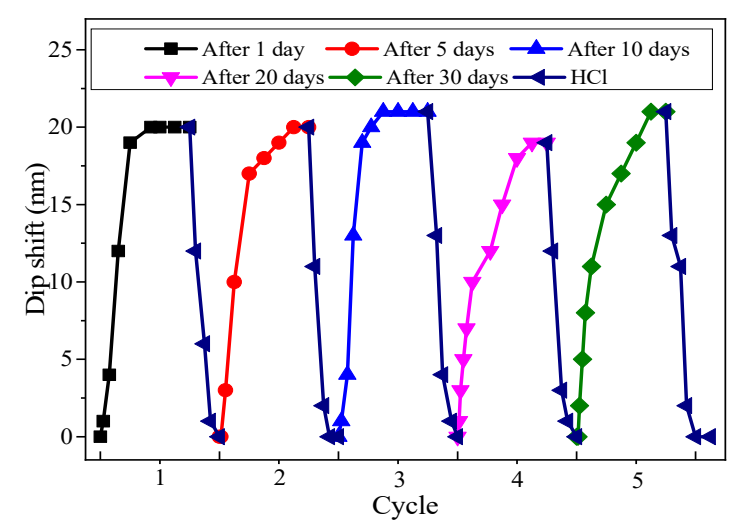

(b)

Figure 11. Repetitive experiment of the SPR $\mathrm{Hg}^{2+}$ sensor in different $\mathrm{Hg}^{2+}$ concentrations (a) and the same $\mathrm{Hg}^{2+}$ concentration $(\mathbf{b})$. 


\section{Conclusions}

In this paper, a reflective fiber SPR sensor based on an MMF-SMF structure was proposed to measure the concentration of $\mathrm{Hg}^{2+}$ in solution. We spliced SMF and MMF, coated Au as an SPR metal film, and attached CS and PAA as a mercury ion sensitive film to form a sensor probe. The experiment results showed that the detection sensitivity of the sensor was up to $0.5586 \mathrm{~nm} / \mu \mathrm{M}$. Moreover, the sensor had a certain specificity and repeatability, and the performance did not change significantly within one month. This reflective sensing structure is good for remote detection of metal ions and can be further used for measuring the concentration of other metal ions if suitable sensitive materials are selected.

Author Contributions: The presented work is a result of the intellectual contribution of the whole team. Z.C. designed the sensor structure and wrote the manuscript; K.H. performed the experiments and analyzed the experimental results; Y.-N.Z. guided the experiments and gave some advice. All authors contributed and approved the final manuscript.

Funding: This research was funded by National Natural Science Foundation of China, grant number 61703080 and 61773102, Fundamental Research Fund for the Central Universities under Grant N180404011, State Key Laboratory of Synthetical Automation for Process Industries under Grant 2013ZCX09, and Scientific Project of Undergraduate under grant 180151.

Conflicts of Interest: The authors declare no conflict of interest.

\section{References}

1. Han, G.R.; Jang, C.H. Detection of heavy-metal ions using liquid crystal droplet patterns modulated by interaction between negatively charged carboxylate and heavy-metal cations. Talanta 2014, 128, 44-50. [CrossRef]

2. Gracia, R.C.; Snodgrass, W.R. Lead toxicity and chelation therapy. Am. J. Health Syst. Pharm. 2007, 64, 45-53. [CrossRef] [PubMed]

3. Rice, K.M., Jr.; Walker, E.M.W.; Wu, M.Z.; Gilette, C.; Blough, E.R. Environmental mercury and its toxic effects. J. Prev. Med. Public Health 2014, 47, 74-83. [CrossRef]

4. Vázquez-González, M.; Carrillo-Carrion, C. Analytical strategies based on quantum dots for heavy metal ions detection. J. Biomed. Opt. 2014, 19, 101503. [CrossRef]

5. Wang, Z.; Lee, J.H.; Lu, Y. Highly sensitive "turn-on" fluorescent sensor for $\mathrm{Hg} 2+$ in aqueous solution based on structure-switching DNA. Chem. Commun. 2008, 45, 6005-6007. [CrossRef]

6. Yang, Y.; Yook, K.; Tae, J. A rhodamine-based fluorescent and colorimetric chemodosimeter for the rapid detection of Hg2+ ions in aqueous media. J. Am. Chem. Soc. 2005, 127, 16760-16761. [CrossRef]

7. Liu, C.W.; Hsieh, Y.T.; Huang, C.C.; Lin, Z.H.; Chang, H.T. Detection of mercury (II) based on Hg2+-DNA complexes inducing the aggregation of gold nanoparticles. Chem. Commun. 2008, 45, 2242-2244. [CrossRef] [PubMed]

8. Huang, X.; Meng, J.; Dong, Y.; Cheng, Y.; Zhu, C. Polymer-based fluorescence sensors incorporating chiral binaphthyl and benzo[2,1,3] thiadiazole moieties for Hg2+ detection. J. Polym. Sci. Pol. Chem. 2010, 48, 997-1006. [CrossRef]

9. Zhang, Y.N.; Zhou, T.; Han, B.; Zhang, A.; Zhao, Y. Optical bio-chemical sensors based on whispering gallery mode resonators. Nanoscale 2018, 10, 13832-13856. [CrossRef]

10. Vargas-Rodriguez, E.; Guzman-Chavez, A.; Cano-Contreras, M.; Gallegos-Arellano, E.; Jauregui-Vazquez, D.; Hernández-García, J.; Estudillo-Ayala, J.M.; Rojas-Laguna, R. Analytical modelling of a refractive index sensor based on an intrinsic micro Fabry-Perot interferometer. Sensors 2015, 15, 26128-26142. [CrossRef] [PubMed]

11. Zhou, J.; Wang, Y.; Liao, C.; Sun, B.; He, J.; Yin, G.; Liu, S.; Li, Z.; Wang, G.; Zhong, X.; et al. Intensity modulated refractive index sensor based on optical fiber Michelson interferometer. Sens. Actuators B 2015, 208, 315-319. [CrossRef]

12. Long, F.; Zhu, A.; Shi, H.; Wang, H.; Liu, J. Rapid on-site/in-situ detection of heavy metal ions in environmental water using a structure-switching DNA optical biosensor. Sci. Rep. 2009, 5, 224-226. [CrossRef] [PubMed] 
13. Zhang, Y.N.; Zhang, L.; Han, B.; Gao, P.; Wu, Q.; Zhang, A. Reflective mercury ion and temperature sensor based on a functionalized no-core fiber combined with a fiber Bragg grating. Sens. Actuators B Chem. 2018, 272, 331-339. [CrossRef]

14. Tan, H.; Zhang, Y.; Chen, Y. Detection of mercury ions(Hg2+) in urine using a terbium chelate fluorescent probe. Sens. Actuators B Chem. 2011, 156, 120-125. [CrossRef]

15. Coelho, L.; de Almeida, J.M.M.M.; Santos, J.L.; Ferreira, R.A.S.; André, P.S.; Viegas, D. Sensing structure based on surface plasmon resonance in chemically etched single mode optical fibres. Plasmonics 2015, 10, 319-327. [CrossRef]

16. Tabassum, R.; Gupta, B.D. Fiber optic manganese ions sensor using SPR and nanocomposite of ZnO-polypyrrole. Sens. Actuators B Chem. 2015, 220, 903-909. [CrossRef]

17. Verma, R.; Gupta, B.D. Detection of heavy metal ions in contaminated water by surface plasmon resonance based optical fibre sensor using conducting polymer and chitosan. Food Chem. 2015, 166, 568-575. [CrossRef]

18. Raj, D.R.; Prasanth, S.; Vineeshkumar, T.V.; Sudarsanakumar, C. Surface plasmon resonance based fiber optic sensor for mercury detection using gold nanoparticles PVA hybrid. Opt. Commun. 2016, 367, 102-107.

19. Jia, S.; Bian, C.; Sun, J.; Tong, J.; Xia, S. A wavelength-modulated localized surface plasmon resonance (LSPR) optical fiber sensor for sensitive detection of mercury (II) ion by gold nanoparticles-DNA conjugates. Biosens. Bioelectron. 2018, 114, 15-21. [CrossRef]

20. Raghunandhan, R.; Chen, L.H.; Long, H.Y.; Leam, L.L.; So, P.L.; Ning, X.; Chan, C.C. Chitosan/PAA based fiber-optic interferometric sensor for heavy metal ions detection. Sens. Actuators B Chem. 2016, 233, 31-38. [CrossRef]

21. Hosoki, A.; Nishiyama, M.; Igawa, H.; Watanabe, K. Multipoint hydrogen sensing of hetero-core fiber SPR tip sensors with pseudorandom noise code correlation reflectometry. IEEE Sens. J. 2016, 16, 2447-2452. [CrossRef]

22. Haddouche, I.; Cherbi, L.; Ferhat, M.L. Analytical modelization of a fiber optic-based surface plasmon resonance sensor. Opt. Commun. 2017, 402, 618-623. [CrossRef]

23. Velázquez-González, J.S.; Monzón-Hernández, D.; Moreno-Hernández, D.; Martínez-Piñón, F.; Hernández-Romano, I. Simultaneous measurement of refractive index and temperature using a SPR-based fiber optic sensor. Sens. Actuators B Chem. 2017, 242, 912-920. [CrossRef]

24. Yang, X.; Lu, Y.; Liu, B.; Yao, J. Simultaneous measurement of refractive index and temperature based on SPR in D-shaped MOF. Appl. Opt. 2017, 56, 4369-4374. [CrossRef] 